\title{
“Obtención De Compost A Partir De Residuos Sólidos Orgánicos Generados En El Mercado Mayorista Del Cantón Riobamba"
}

\author{
Brito Hanníbal \\ Docente Investigador Grupo de Investigación Ambiental y Desarrollo de la \\ ESPOCH (GIADE) \\ Viteri Rafaela \\ Guevara Luis \\ Villacrés Mario \\ Jara Janeth \\ Docente Investigador Facultad de Ciencias
}

Jiménez Silvio

Colaborador Facultad de Ciencias

Moya Paola
Parra Carina

Colaborador GADIPM Cayambe

doi: 10.19044/esj.2016.v12n29p76 URL:http://dx.doi.org/10.19044/esj.2016.v12n29p76

\begin{abstract}
The process of obtaining compost was developed at the Agroambiental Ricpamba theme park administrated by the Autonomous Municipal Government of Riobamba (GADM, where an organic fertilizer was obtained from solid waste generated in the wholesale market of the city of Riobamba in order to be use in parks, gardens, green spaces and in agriculture, for which, a stack of 1,0 tonne was armed formed with fixed proportions in relation to weight / weight from three organic components of plant type: Organic solid waste wholesale market $(600 \mathrm{~kg})$, tree pruning waste of the Polytechnic School of Chimborazo (300 kg), and prunings waste of ornamental palm (Phoenix canariensis) from the city of Riobamba (100 $\mathrm{kg}$ ) To achieve adequate carbon nitrogen ratio $(\mathrm{C} / \mathrm{N})$. The compost was performed using an open stack system and manual turning; during the process a daily control of temperature and humidity was performed, besides that during each dump material samples were taken for analysis of the most important parameters, thus controlling the process. The temperature evolution of the stack in the mesophilic phase reached $45{ }^{\circ} \mathrm{C}$ and thermophilic phase temperature was above $60{ }^{\circ} \mathrm{C}$, which was controlled with
\end{abstract}


manual turns. Once the process was finished the quality of the compost was proved by the physical-chemical analysis, chemical-biological to determine its quality. Additionally the solid waste generated in the Public Municipal Market of "San Pedro de Riobamba" (EP-EMMPA) were classified and quantified on a daily basis. The solid waste were classified in 6 groups: organic, plastics, paper, cardboard, wood and others, being organic waste the ones found in a greater amount, in an average of $95.59 \%$. The obtained product has the right physical-chemical, chemical-biological property to be used as an organic fertilizer or soil improver by providing essential nutrients, macro and micro elements required by plants.

Keywords: Compost, Organic Fertilizer, Composting, Organic Matter, Local Markets, Recycling, Solid Waste

\section{Resumen}

La obtención de compost se desarrolló en el parque Temático Agroambiental Ricpamba administrado por el Gobierno Autónomo Descentralizado Municipal (GADM) de Riobamba, donde se obtuvo un abono orgánico a partir de los residuos sólidos generados en el Mercado Mayorista de la ciudad de Riobamba para su uso en parques, jardines, espacios verdes y el aprovechamiento en agricultura, para lo cual, se armó una pila de 1,0 tonelada constituida con proporciones fijas en relación peso/peso de tres componentes orgánicos de tipo vegetal: residuos sólidos orgánicos del mercado Mayorista $(600 \mathrm{~kg})$, residuos de poda de árboles de la Escuela Superior Politécnica de Chimborazo $(300 \mathrm{~kg})$ y residuos de poda de palma ornamental (Phoenix canariensis) de la ciudad de Riobamba (100 kg) para lograr una adecuada relación de carbono nitrógeno $(\mathrm{C} / \mathrm{N})$. El compost se realizó mediante un sistema de pila abierta y volteo manual; durante el proceso se realizó un control diario de temperatura y humedad, además durante cada volteo se tomó muestras de material para realizar análisis de los parámetros más importantes, de esta manera controlar el proceso. La evolución de la temperatura de la pila en la fase mesófila alcanzó los $45^{\circ} \mathrm{C}$ y en la fase termófila la temperatura fue superior a $60{ }^{\circ} \mathrm{C}$, la misma que se controló con volteos manuales. Una vez finalizado el proceso se comprobó la calidad del compost mediante análisis físicos-químicos, químicos $\mathrm{y}$ biológicos para determinar su calidad. De forma adicional se clasificaron y cuantificaron los residuos sólidos generados en la Empresa Pública Municipal Mercado de Productores Agrícolas "San Pedro de Riobamba" (EP-EMMPA) en los diferentes días de actividad comercial. Los residuos sólidos se clasificaron en 6 grupos: orgánicos, plásticos, papel, cartón, madera y otros, siendo los residuos orgánicos los que encuentran mayor 
cantidad presentando un promedio total del $95,59 \%$. El producto obtenido posee las propiedades físico-químicas, químicas y biológicas adecuadas para ser empleado como abono orgánico o mejorador de suelos mediante el aporte de nutrientes esenciales, macroelementos y microelementos requeridos por las plantas.

Palabras clave: Compost, Abono Orgánico, Compostaje, Materia Orgánica, Mercados Municipales, Reciclaje, Residuos Sólidos

\section{Introducción}

El compostaje es un proceso biotecnológico en el que se producen reacciones bioquímicas, para transformar la fracción orgánica mediante la actividad de organismos aerobios como poblaciones de bacterias, hongos y actinomicetos, en un producto final estable, libre de semillas y patógenos denominado compost. Éste es un producto apreciado por contener un alto porcentaje de sustancias húmicas que es utilizado como un mejorador del suelo, abono orgánico y como parte de sustratos de cultivo para semilleros. Todos los materiales orgánicos de origen vegetal o animal, que en su composición contengan una gran cantidad de materia orgánica biodegradable se puede utilizar para elaborar compost.

El proceso de compostaje es una tecnología con costos muy bajos en la que se logra transformar los residuos y varios subproductos orgánicos en productos que sirven como enmienda o abonos para los suelos, lo cual permite reducir los impactos ambientales y mejorar las propiedades de los suelos, además permite aprovechar los residuos orgánicos y obtener productos que pueden ser fácilmente manejados y almacenados, en la que su materia orgánica es muy estable, humificada y libre de sustancias toxicas, patógenos y semillas de malas hierbas.

El presente trabajo hace referencia a la elaboración de compost a partir de los residuos sólidos orgánicos generados en la Empresa Pública Municipal Mercado de Productores Agrícolas "San Pedro de Riobamba" (EP-EMMPA) los mismos que durante muchos años y hasta la actualidad son depositados en el botadero a cielo abierto de Porlón del cantón Riobamba sin contar con un sistema de gestión previo para su manejo apropiado. Esta gestión inadecuada trae consigo problemas sociales y ambientales, que atentan contra el ambiente y la salud de las personas como consecuencia de la proliferación de enfermedades trasmitidas por vectores, la contaminación del aire, agua, suelo, gases efecto invernadero y la generación de lixiviados.

Para la elaboración del compost se tomó $600 \mathrm{~kg}$ de residuos sólidos (OLIVEIRA THAIS, 2015) orgánicos de mercado y se mezclaron con 300 $\mathrm{kg}$ de residuos secos de poda de árboles y $100 \mathrm{~kg}$ de poda de palma 
ornamental con los cuales se formó una pila de $1000 \mathrm{~kg}$ aproximadamente. Los componentes de la pila fueron triturados previamente con un molino de cuchillas. El proceso de compostaje se realizó mediante volteo manual a cielo abierto, el control de los parámetros se realizó mediante datos obtenidos en el trabajo de campo como en los resultados de las muestras analizadas en el laboratorio para determinar el avance del proceso y la calidad del producto.

Los objetivos de este trabajo están enfocados en la obtención de compost a partir de los residuos sólidos de mercado para obtener un producto con las propiedades físico-químicas, químicas y biológicas que brinden las características de un producto óptimo para ser utilizado en agricultura.

\section{Materiales y métodos \\ Preparación del terreno}

Con la ayuda de una mini cargadora Caterpillar se realizó un terraplén con espacio suficiente para realizar el proceso de compostaje, una vez realizado el terraplén, se delimitaron dos áreas de $2 \mathrm{~m} \times 3 \mathrm{~m}$ para realizar los volteos, para evitar la contaminación por contacto directo con el suelo se colocó una capa de material vegetal entre el suelo y la pila, para realizar el volteo en la segunda área que se estableció para tal fin nuevamente se colocó una capa vegetal en donde era transpuesta la pila, este proceso se repitió hasta finalizar los volteos.

\section{Preparación del material orgánico}

Los residuos secos de poda de árboles se tomaron de varios montículos los mismos que estaban dispuestos en una área dentro de la Escuela Superior politécnica de Chimborazo que es destinada para depositar este tipo de desechos, los residuos de poda de palma se transportaron y se depositaron en el área destinada para el trabajo dentro del parque Ricpamba, en cuanto a los residuos orgánicos generados en el mercado mayorista estos se recolectaron en sacos de plástico de los recipientes recolectores dispuestos en las diferentes secciones de comercio, puestos de venta y de los contenedores donde se almacena gran cantidad de los residuos del mercado y se transportaron al parque Ricpamba.

Una vez obtenido los tres componentes, con un molino de cuchillas los residuos de árboles y palma fueron triturados hasta obtener un tamaño de partícula inferior a $1,8 \mathrm{~cm}$ en tanto que los residuos de mercado fueron inferior a $5 \mathrm{~cm}$, los residuos fueron triturados hasta obtener aproximadamente $300 \mathrm{~kg}$ de residuos de árboles, $100 \mathrm{~kg}$ de residuos de palma y $600 \mathrm{~kg}$ de desechos de mercado (SANABRIA. M, 2013, pág. 23). 


\section{Implantación de la pila}

Una vez establecida el área de trabajo, los materiales orgánicos de mercado se mezclaron con los materiales secos, para ello se usó palas manuales hasta lograr una combinación homogénea de los residuos a ser compostados formando una pila con forma trapezoidal, de $2 \mathrm{~m}$ de ancho, $3 \mathrm{~m}$ de largo, $1 \mathrm{~m}$ de alto y un peso aproximado de 1,0 tonelada. La mezcla homogénea quedo establecida con un porcentaje de humedad del 46,4\% valor que está dentro de lo establecido por la (FAO, 2013, pág. 27) que recomienda un porcentaje de humedad entre $45 \%$ y $60 \%$ como adecuado para elaborar compost.

\section{Registro de la temperatura y humedad}

Una vez conformada la pila el control de la temperatura y humedad se realizó diariamente durante la primera semana y después una vez por semana, para medir estos dos parámetros se establecieron 14 puntos de muestreos en la pila, 3 puntos en cada lado mayor de la pila, 2 puntos en cada lado menor de la pila y 4 puntos en la parte superior de la misma, para medir la temperatura se utilizó un termómetro digital portátil en forma de (T) modelo HI 145 con una precisión $\pm 0,3{ }^{\circ} \mathrm{C}$ que alcanza a medir la temperatura a una profundidad de $30 \mathrm{~cm}$ y para medir la humedad se utilizó un higrómetro que toma medidas a una profundidad de $20 \mathrm{~cm}$ de profundidad. La frecuencia de riego dependió de la precipitación de la zona.

\section{Realización de volteos}

Los volteos se realizaron de forma manual para lo cual se utilizaron palas, el material en descomposición fue traspuesto en una área adyacente a la pila y para completar el volteo era traído a su posición inicial. Los primeros volteos se realizaron cada 14 días durante dos meses para homogenizar el material, para posterior a ello solo cuando la temperatura medida a una profundidad de $30 \mathrm{~cm}$ alcanzó valores inferiores a los $30{ }^{\circ} \mathrm{C}$. Los volteos de la pila se realizaron hasta que la temperatura de la misma alcanzó un valor cercano a la temperatura ambiente promedio que varía entre $12{ }^{\circ} \mathrm{C}$ y $15^{\circ} \mathrm{C}$ según los registros de la estación meteorológica (ESPOCH, 2015) una vez alcanzado esta condición se dieron por terminado los volteos para permitir que el proceso entre a su etapa de maduración y finalizar el proceso de compostaje.

\section{Toma de muestras}

La pila se armó el 01 de diciembre del 2014. El compost se obtuvo después de 224 días después que se inició el proceso, la fase degradativa tuvo un tiempo de duración de 168 días dándose por terminado el 05 de mayo del 2015, para posteriormente a ello entrar a la fase de maduración que 
duró 56 días debido a las bajas temperaturas del material, tiempo superior a lo establecido como óptimo en la fase de maduración que dura aproximadamente ente 20 días según (JARAMILLO. G \& ZAPATA. L, 2008, pág. 36), para dar por finalizado el proceso el 13 de julio del 2015.

\section{Refinado}

Concluido el proceso de maduración, se procedió a una clasificación física del material mediante un tamiz para desechar todos los elementos gruesos para lo cual se utilizó una malla con orificios cuadrados de $0,5 \mathrm{~cm}$ de diámetro lo cual nos permitió aislar todo el material no deseado como una forma de estimar la calidad del producto, el tamaño de partícula del material es el adecuado puesto que un producto de calidad presenta un tamaño de partícula inferior a 1,6 cm según la (FAO, 2013, pág. 31).

\section{Determinación del rendimiento del proceso}

Todo material se colocó en sacos de plástico tanto el abono como el material rechazado por diferencia de peso se determinó el rendimiento. Se tomó en cuenta el peso del material extraído para muestreos.

\section{Preparación de las muestras para realizar análisis en el laboratorio}

Durante cada volteo se extrajo una muestra de $2 \mathrm{~kg}$, la misma que fue llevada al laboratorio y secada en un secador de bandejas a $70{ }^{\circ} \mathrm{C}$ por un tiempo de 24 horas, de este material se tomó una submuestra de $250 \mathrm{~g}$ posteriormente el material fue triturado con un molino hasta obtener un tamaño de partícula de $3 \mathrm{~mm}$, este material con la ayuda de un mortero se trituro nuevamente hasta obtener un tamaño de partícula de $425 \mu \mathrm{m}$ aproximadamente, una vez realizado este procedimiento el material fue colocado en fundas ziploc y almacenadas hasta su posterior uso.

\section{Determinación de la conductividad eléctrica y pH}

Del material preparado se pesó $5 \mathrm{~g}$ de la muestra y colocó en un vaso de precipitación de $100 \mathrm{~mL}$, se añadió $60 \mathrm{~mL}$ de agua destilada, se agitó por 10 min y se dejó reposar por 10 min hasta su total homogenización, luego se introdujo los electrodos del instrumento en la solución acuosa y se midió la conductividad eléctrica. Para medir el $\mathrm{pH}$ se retiró los electrodos del instrumento, se lavó con agua destilada y nuevamente se introdujo en la solución y se realizó la medición del pH.

\section{Determinación del índice de germinación}

De la muestra seca, se hizo una extracción acuosa, relación 1:20 (p/v) agitando mecánicamente durante 2 horas, luego se centrifugó a $7000 \mathrm{rpm}$ 
durante $10 \min$ y se filtró con papel de $0,45 \mu \mathrm{m}$ mediante vacío para esterilizar el extracto, este extracto se colocó en placas Petri de $10 \mathrm{~cm}$ de diámetro, cubiertas con papel filtro, se colocaron 10 semillas de rábano y añadió $10,0 \mathrm{~mL}$ del extracto acuoso mojando el papel en su totalidad, realizando 10 repeticiones, para preparar la muestra blanco se utilizó agua destilada, una muestra por cada repetición. Una vez preparadas las cajas con las muestras del extracto y blanco estas fueron envueltas con papel aluminio haciendo grupos de 5 unidades para evitar resequedad y brindar oscuridad, cada grupo de cajas se colocó en la estufa de incubación de forma aleatoria a $80 \%$ de humedad, $22{ }^{\circ} \mathrm{C}$ y por un tiempo de 120 horas, cumplido este tiempo se extrajo las cajas con las semillas y se cuantificó el número de semillas germinadas y la longitud alcanzada por las raíces por placa, finalmente se determinó el índice de germinación.

\section{Resultados y discusión}

Tabla 1: Resultado de los componentes iniciales

\begin{tabular}{|c|c|c|c|c|}
\hline $\begin{array}{c}\text { PARÁMETRO } \\
\text { ANALIZADO }\end{array}$ & \multicolumn{3}{|c|}{ MUESTRAS } & $\begin{array}{c}\text { UNIDAD DE } \\
\text { MEDIDA }\end{array}$ \\
\hline & Árboles & Palma & RSO & \\
\hline *Cenizas & 7,79 & 32,21 & 14,05 & $\%$ \\
\hline Conductividad Eléctrica & 8,09 & 4,02 & 6,68 & $\mathrm{mS}$ \\
\hline Humedad & 9,11 & 13,37 & 82,24 & $\%$ \\
\hline *M. Orgánica & 92,21 & 67,79 & 85,95 & $\%$ \\
\hline pH & 5,23 & 5,80 & 8,28 & Unidades \\
\hline Relación C/N & 22,47 & 43,34 & 25,67 & Unidades \\
\hline *Calcio & 1,67 & 1,62 & 1,16 & $\%$ \\
\hline Carbono & 50,11 & 36,84 & 46,71 & $\%$ \\
\hline$*$ Cobre & 6,39 & 28,08 & 9,49 & $\mathrm{ppm}$ \\
\hline *Fósforo & 0,1 & 0,1 & 0,2 & $\%$ \\
\hline *Hierro & 289,4 & 4400,9 & 1063,0 & $\mathrm{ppm}$ \\
\hline *Magnesio & 0,42 & 0,39 & 0,25 & $\%$ \\
\hline *Manganeso & 26,78 & 66,15 & 52,55 & $\mathrm{ppm}$ \\
\hline *Nitrógeno & 2,23 & 0,85 & 1,82 & $\%$ \\
\hline *Potasio & 0,55 & 0,80 & 1,88 & $\%$ \\
\hline *Zinc & 14,39 & 52,66 & 29,37 & $\mathrm{ppm}$ \\
\hline
\end{tabular}

Tabla 2: Resultado del control del proceso

\begin{tabular}{|c|c|c|c|c|c|c|c|c|c|c|c|}
\hline \multirow{2}{*}{$\begin{array}{c}\text { PARÁMETRO } \\
\text { ANALIZADO }\end{array}$} & $\mathrm{P}_{\mathrm{m}} \mathrm{T}_{0}$ & $\begin{array}{c}\mathrm{P}_{\mathrm{m}} \mathrm{T} \\
1\end{array}$ & $\begin{array}{c}\mathrm{P}_{\mathrm{m}} \mathrm{T} \\
2\end{array}$ & $\begin{array}{c}\mathrm{P}_{\mathrm{m}} \mathrm{T} \\
3\end{array}$ & $\begin{array}{c}\mathrm{P}_{\mathrm{m}} \mathrm{T} \\
4\end{array}$ & $\begin{array}{c}\mathrm{P}_{\mathrm{m}} \mathrm{T} \\
5\end{array}$ & $\begin{array}{c}\mathrm{P}_{\mathrm{m}} \mathrm{T} \\
6\end{array}$ & $\begin{array}{c}\mathrm{P}_{\mathrm{m}} \mathrm{T} \\
7\end{array}$ & $\begin{array}{c}\mathrm{P}_{\mathrm{m}} \mathrm{T} \\
8\end{array}$ & $\mathrm{P}_{\mathrm{m}} \mathrm{T}_{9}$ & $\begin{array}{c}\text { UNIDAD } \\
\text { DEDIDA }\end{array}$ \\
\hline $\begin{array}{c}\text { Tiempo de } \\
\text { obtención }\end{array}$ & 0 & 14 & 28 & 42 & 56 & 98 & 126 & 154 & 168 & 224 & días \\
\hline *Cenizas & 13,97 & $\begin{array}{c}29,0 \\
7\end{array}$ & $\begin{array}{c}32,6 \\
7\end{array}$ & $\begin{array}{c}39,6 \\
2\end{array}$ & $\begin{array}{c}42,4 \\
7\end{array}$ & $\begin{array}{c}45,3 \\
2\end{array}$ & $\begin{array}{c}47,3 \\
5\end{array}$ & $\begin{array}{c}47,9 \\
7\end{array}$ & $\begin{array}{c}48,8 \\
9\end{array}$ & 50,41 & $\%$ \\
\hline C. Eléctrica & 2,10 & 2,07 & 2,00 & 1,96 & 1,95 & 2,10 & 2,22 & 2,10 & 2,00 & 2,20 & $\mathrm{mS} / \mathrm{cm}$ \\
\hline *M. Orgánica & 86,03 & 70,9 & 67,3 & 60,3 & 57,5 & 54,6 & 52,6 & 52,0 & 51,1 & 49,59 & $\%$ \\
\hline
\end{tabular}




\begin{tabular}{|c|c|c|c|c|c|c|c|c|c|c|c|}
\hline & & 3 & 3 & 8 & 3 & 8 & 5 & 3 & 1 & & \\
\hline $\mathrm{pH}$ & 6,50 & 7,10 & 7,75 & 8,25 & 8,50 & 8,67 & 8,70 & 8,75 & 8,80 & 8,85 & Unidades \\
\hline Relación C/N & 25,83 & & & & & & & & & 12,25 & Unidades \\
\hline$*$ Calcio & 1,60 & & & & & & & & & & $\%$ \\
\hline Carbono & 46,76 & $\begin{array}{c}38,5 \\
5\end{array}$ & $\begin{array}{c}36,5 \\
9\end{array}$ & $\begin{array}{c}32,8 \\
2\end{array}$ & $\begin{array}{c}31,2 \\
7\end{array}$ & $\begin{array}{c}29,7 \\
2\end{array}$ & $\begin{array}{c}28,6 \\
2\end{array}$ & $\begin{array}{c}28,2 \\
8\end{array}$ & $\begin{array}{c}27,7 \\
8\end{array}$ & 26,95 & $\%$ \\
\hline *Cobre & 43,57 & & & & & & & & & & ppm \\
\hline *Fósforo & 0,10 & 0,11 & 0,13 & 0,15 & 0,17 & 0,20 & 0,23 & 0,26 & 0,30 & 0,34 & $\%$ \\
\hline$*$ Hierro & 2476,0 & & & & & & & & & & ppm \\
\hline *Magnesio & 0,23 & & & & & & & & & & $\%$ \\
\hline *Manganeso & 38,47 & & & & & & & & & & ppm \\
\hline *Nitrógeno & 1,81 & & & & & & & & & 2,20 & $\%$ \\
\hline *Potasio $\left(\mathrm{K}_{2} \mathrm{O}\right)$ & 0,89 & 0,96 & 1,03 & 1,10 & 1,18 & 1,27 & 1,36 & 1,47 & 1,57 & 1,69 & $\%$ \\
\hline$*$ Zinc & 46,86 & & & & & & & & & & $\mathrm{ppm}$ \\
\hline Color & $\begin{array}{l}\text { Pardo-- } \\
\text { claro }\end{array}$ & & & & & & & & & $\begin{array}{l}\text { Pardo- } \\
\text { oscuro }\end{array}$ & - \\
\hline $\begin{array}{l}\text { Densidad } \\
\text { aparente }\end{array}$ & 392,57 & $\begin{array}{c}404, \\
99\end{array}$ & $\begin{array}{c}427 \\
61\end{array}$ & $\begin{array}{c}441 \\
46\end{array}$ & $\begin{array}{c}466 \\
06\end{array}$ & $\begin{array}{c}481 \\
40\end{array}$ & $\begin{array}{c}508 \\
29\end{array}$ & $\begin{array}{c}525 \\
18\end{array}$ & $\begin{array}{c}554 \\
47 \\
\end{array}$ & 573,14 & $\mathrm{~kg} / \mathrm{m}^{3}$ \\
\hline Olor & $\begin{array}{c}\text { Amoniac } \\
\mathrm{o}\end{array}$ & & & & & & & & & $\begin{array}{l}\text { Tierra } \\
\text { fresca }\end{array}$ & - \\
\hline $\begin{array}{c}\text { Tamaño de } \\
\text { partícula }\end{array}$ & Variado & & & & & & & & & $<1$ & $\mathrm{~cm}$ \\
\hline $\begin{array}{l}\text { Indice de } \\
\text { germinación }\end{array}$ & 48,76 & & & & & & & & & 85,25 & $\%$ \\
\hline
\end{tabular}

Tabla 3: Resultado del control del proceso

\begin{tabular}{|c|c|c|c|c|}
\hline COMPONENTES & TESTIGO & $\mathrm{P}_{\mathrm{m}} \mathrm{T}_{0}$ & $\mathrm{P}_{\mathrm{m}} \mathrm{T}_{9}$ & UNIDAD \\
\hline SG & 92,50 & 77,50 & 91,25 & $\%$ \\
\hline SSG & 7,50 & 22,50 & 8,75 & $\%$ \\
\hline GRS & - & 83,78 & 98,65 & $\%$ \\
\hline CRR & - & 58,20 & 86,42 & $\%$ \\
\hline IG & - & 48,76 & 85,25 & $\%$ \\
\hline
\end{tabular}

\section{Donde}

SG:

Semillas germinadas

SSG: $\quad$ Semillas sin germinar

GRS: $\quad$ Germinación relativa de las semillas

CRR: $\quad$ Crecimiento relativo de la raíz

IG: Índice de germinación

$\mathrm{P}_{\mathrm{MT}} \mathrm{T}_{0}$ : Muestra inicial

$\mathrm{P}_{\mathrm{M}} \mathrm{T}_{9}$ : Muestra final 
Tabla 4: Resultado del control del proceso

\begin{tabular}{|c|c|c|c|}
\hline NOMBRE & COMPONENTES & PESO $(\mathrm{kg})$ & PORCENTAJE (\%) \\
\hline \multirow{4}{*}{ Pila de compost } & Peso inicial & 1000,0 & - \\
\cline { 2 - 4 } & Producto final & 540,0 & 54,0 \\
\cline { 2 - 4 } & $\begin{array}{c}\text { Pérdida de peso por } \\
\text { descomposición y volteos }\end{array}$ & 364,0 & 36,4 \\
\cline { 2 - 4 } & $\begin{array}{c}\text { Pérdida de peso por } \\
\text { refinación }\end{array}$ & 76,0 & 7,6 \\
\cline { 2 - 4 } & $\begin{array}{c}\text { Pérdida de peso por } \\
\text { extracción de material para } \\
\text { muestreos }\end{array}$ & 20,0 & 2,0 \\
\hline
\end{tabular}

\section{Discusión}

\section{Caracterización de los sustratos}

En la tabla 1 se muestra los resultados de los análisis que se realizaron a los componente que formaron la pila. Con respecto al $\mathrm{pH}, \mathrm{los}$ residuos de poda de árboles presentaron un valor ligeramente ácido de 5,23 mientras que los residuos orgánicos de mercado tuvieron un valor ligeramente básico de 8,28 , esto probablemente se debe a que sus residuos estaban conformados en gran porcentaje de restos verduras y en poca cantidad de frutas y cítricos, además estos residuos aun no entraron a una etapa de descomposición en la cual se generan ácidos.

En cuanto a la humedad, los residuos de poda de árboles contenía un porcentaje de $9,11 \%$ y la poda de palma $13,37 \%$ como era de esperase, debido a que estos dos componentes fueron obtenidos a partir de residuos secos mientras que los residuos de mercado su humedad fue de 82,24\% debido a que se trataba de residuos frescos de verduras, hortalizas, frutas, restos de comida preparada, entre otros.

El porcentaje más alto de contenido de carbono fue presentado por los residuos de árboles con un valor de 50,11 \% (residuos secos) y el más bajo los residuos de poda de palma con un valor de $36,84 \%$.

El contenido de nitrógeno más alto se encontró en los residuos de árboles con un valor de 2, 23\%, el valor más bajo se reportó en los residuos de poda de palma con un valor de $0,85 \%$. En cuanto al contenido de fósforo, se encontró en los materiales secos valores de $0,1 \%$ y los para los residuos de mercado $0,2 \%$ debido a la presencia de residuos de verduras y hortalizas que son productos que contienen en mayor cantidad este elemento. El contenido más elevado de potasio reportaron los residuos de mercado con un valor de $1,88 \%$ debido a que en este tipo de residuos contenían restos de comida preparada, rechazos de plátano y frutas que se expenden en el mercado y el más bajo los residuos de palma con un valor de $0,43 \%$ AGROCALIDAD, 2015. 


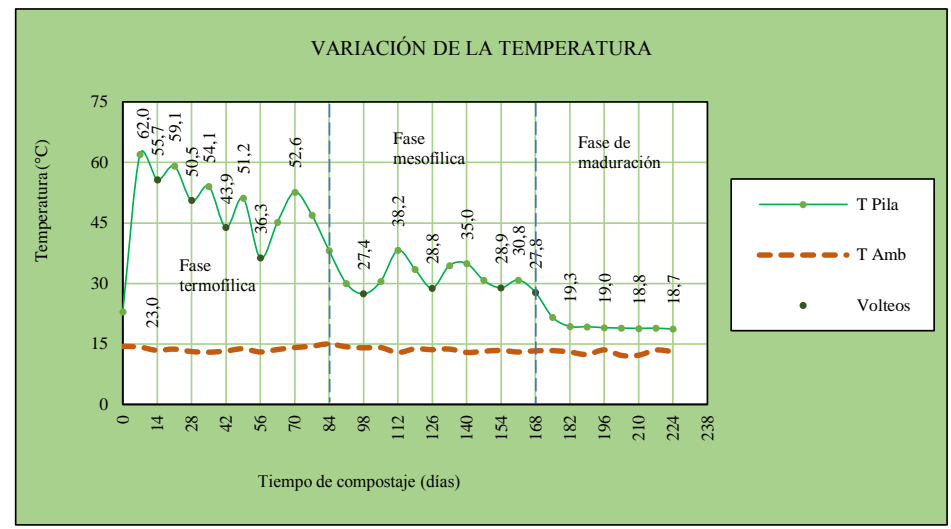

FUENTE: BRITO H. / JIMÉNEZ. S, 2015

Gráfico 1: Variación de la temperatura

Una vez iniciado el proceso de compostaje, se determinó a través de los cambios de temperatura que la etapa de latencia y termofílica inicial fue muy puesto que a partir del día 7 el proceso ya alcanzó la etapa termofílica que marcó temperaturas superiores a los $60{ }^{\circ} \mathrm{C}$ debido a que en la fase mesofílica una intensa actividad metabólica realizada por los microorganismos realizaron la descomposición rápida de los lípidos, glúcidos y proteínas presentes en el medio que aprovecharon la gran cantidad de materia orgánica y sus nutrientes.

La fase termófila y mesófila duraron 84 días, en donde la fase mesófila debido a la trasformación de la materia orgánica alcanzó temperaturas inferiores a $\operatorname{los} 40^{\circ} \mathrm{C}$ en los que parámetros como la humedad, el oxígeno y la temperatura ambiente de la zona no intervinieron como factores limitantes del proceso. Bajo estas de temperatura el material sufrió un proceso de higienización que se considera que se eliminaron organismos patógenos y semillas de malezas puesto que para higienizar el material debe ser expuesto a temperaturas entre 38 y $60{ }^{\circ} \mathrm{C}$ por un tiempo de 15 días (GÓMEZ. Y, González, \& Rubalcaba, 2004, pág. 3). Cumplida estas fases y con temperatura promedio cercana a la ambiente que varía entre 12 y $16^{\circ} \mathrm{C}$, el producto entró en la etapa de maduración que duró 56 días, tiempo necesario para cumplir esta fase puesto que lo recomendado es entre 20 días (JARAMILLO. G \& ZAPATA. L, 2008, pág. 36).

Las temperaturas que se alcanzaron indican que las mezclas de los componentes orgánicos se combinaron en las proporciones adecuadas, así como las dimensiones de la pila también fueron las adecuadas que facilitaron el correcto desarrollo del proceso (MOHEDO. J, 2002, pág. 97). 


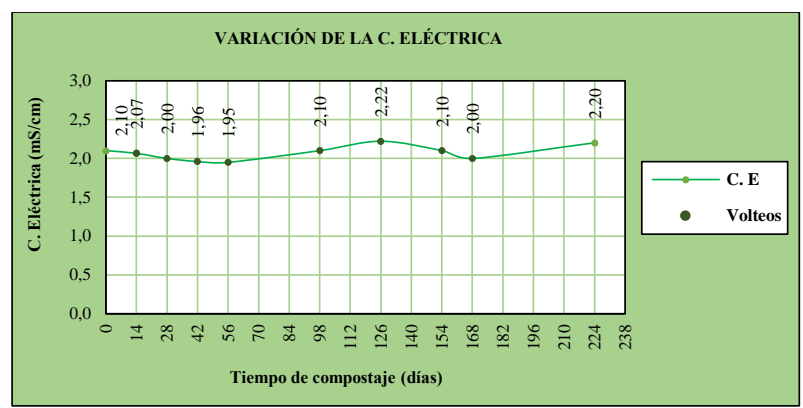

FUENTE: BRITO H. / JIMÉNEZ. S, 2015

Gráfico 2: Variación de la conductividad eléctrica

La CE no varió significativamente como consecuencia de la mineralización de los elementos o por el lavado de las sales formadas debido a la precipitación en la zona o por los riegos realizados para mantener la humedad del material durante el proceso de compostaje. La CE vario en un rango de 1,95 a $2,22 \mathrm{mS} / \mathrm{cm}$. Es preferible que el compost presente valores bajos de CE puesto que la existencia de sales está asociada con la concentración de elementos como el sodio y potasio así como también compuestos de cloruro, nitrato, sulfato y sales de amonio que en concentraciones elevadas inhiben el crecimiento de las plantas. La conductividad eléctrica en nuestro estudio fue de $2,20 \mathrm{mS} / \mathrm{cm}$ valor que está dentro de los establecido para un producto de calidad que es inferior a 3,0 mS/cm (GORDILLO. F \& CHÁVEZ. E, 2010, pág. 4).

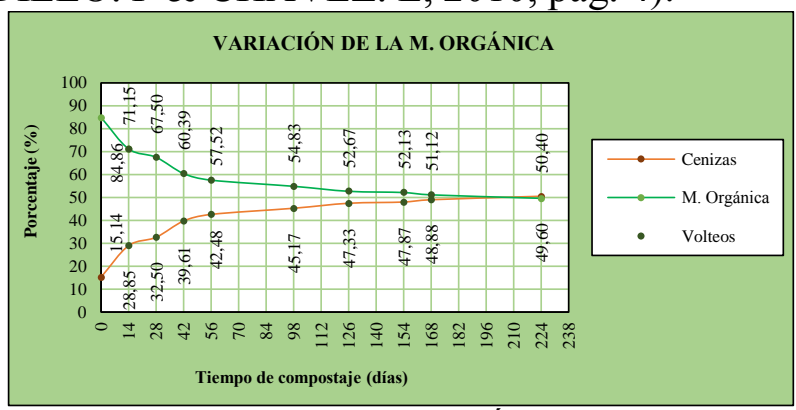

FUENTE: BRITO H. / JIMÉNEZ. S, 2015

Gráfico 3: Variación materia orgánica

El cabio evidente en la pérdida de materia orgánica a lo largo del proceso se debe a que los microorganismos transforman el carbono, principal elemento constituyente de la materia orgánica, en $\mathrm{CO}_{2}$.entre otros compuestos. El porcentaje de materia orgánica que se obtuvo una vez finalizado el proceso fue de 49,59\%. Este valor está dentro de los valores permisibles de materia orgánica que el humus debe tener para ser comercializado el cual se sitúa entre 25 y $70 \%$ (CHÁVEZ. L, 2012, págs. 64-68). 
El resultado obtenido es consecuencia de niveles elevados de materia orgánica presente en los residuos orgánicos de mercado como de los materiales secos, además se debe al alto contenido de celulosa y fibra presentan una estructura muy rígida y poco degradable lo cual influenció en el tiempo de descomposición. Mientras la materia orgánica disminuye, la cantidad de ceniza aumenta en forma proporcional (GALEA. Z, Galea, Camacho,Zesay, 2013, pág. 19).

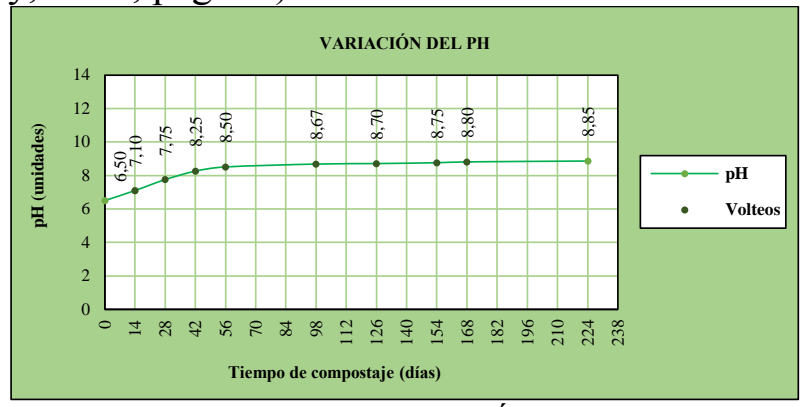

FUENTE: BRITO H. / JIMÉNEZ. S, 2015

Gráfico 4: Variación del $\mathrm{pH}$

La variación de $\mathrm{pH}$ está relacionado con la actividad metabólica de los microorganismos y los nutrientes del medio, el $\mathrm{pH}$ de la materia en descomposición disminuye en la etapa mesofílica como consecuencia de la actividad metabólica de los microorganismos por la descomposición rápida de los lípidos y glúcidos en ácidos pirúvicos y de proteínas en aminoácidos, el pH aumento de 6,50 a 8,85 debido a la degradación de estos ácidos en la etapa termofílica. A demás este incremento de $\mathrm{pH}$ se produjo por la descomposición del nitrógeno presente en el material orgánico formándose amoniaco $\left(\mathrm{NH}_{3}\right)$ que por la presencia de la humedad se diluye formando amonio $\left(\mathrm{NH}_{4}\right)$ (MARMOLEJO. L, OVIEDO. E, JAIMES. J, \& TORRES. P, 2010, págs. 324,325).

Los valores de $\mathrm{pH}$ obtenidos durante el proceso y al final del mismo se consideran como óptimos puesto que tanto el valor mínimo y máximo que presentó la pila están dentro de los rangos permisibles según los valores determinados (ALTAMIRANO. M \& CABRERA. C, 2006, pág. 80), que considera que valores de $\mathrm{pH}$ entre 6 a 9 son óptimos en el proceso de compostaje.

\section{Variación de la relación $\mathbf{C} / \mathbf{N}$}

El carbono disminuye por la pérdida de masa durante el proceso como $\mathrm{CO}_{2}$ o como producto de las reacciones de biooxidación, el nitrógeno aumenta como consecuencia de la pérdida de materia orgánica aunque también se puede perder en forma de óxidos de nitrógeno. Como consecuencia de la transformación de estos elementos la relación $\mathrm{C} / \mathrm{N}$ 
disminuyó desde de 25,83 hasta 12,25. Varios autores indican que la relación óptima $\mathrm{C} / \mathrm{N}$ para un compost maduro es inferior a 20 , en dependencia de la naturaleza de los materiales iniciales. (QUISHPE. D \& SUQUILANDA. M, 2008, pág. 7), como una forma de evaluar la calidad del humus elaborado en el proceso de compostaje se procedió a una realizar análisis del producto final.

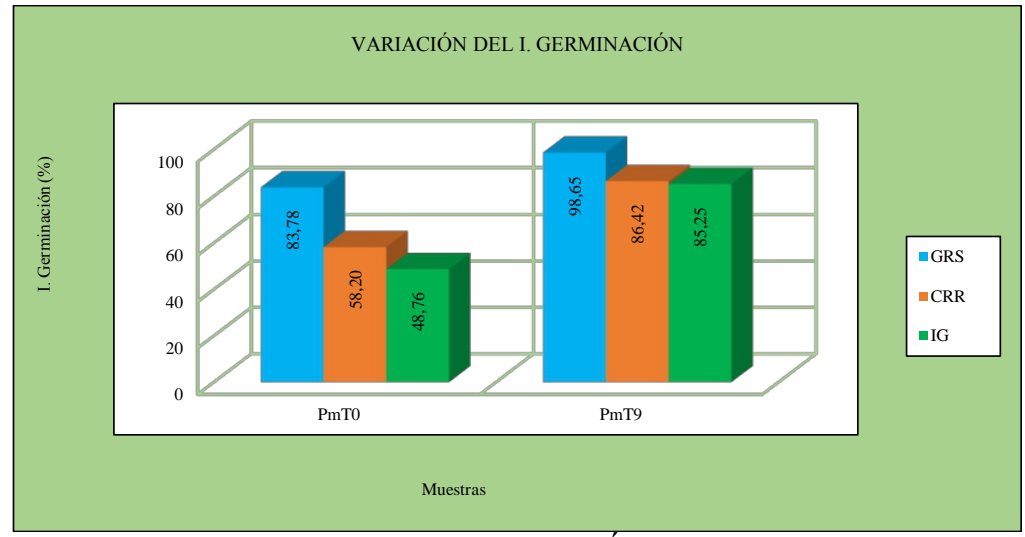

FUENTE: BRITO H. / JIMÉNEZ. S, 2015

Gráfico 5: Índice de germinación y elongación radical

\section{Donde}

$\begin{array}{llll}\text { GRS: } & \text { Germinación relativa } & \text { de } & \text { semillas } \\ \text { CRR: } & \text { Crecimiento relativo de } & & \\ \text { IG: } & \text { Índice de germinación } & & \end{array}$

El porcentaje de germinación del humus obtenido es de 85,25\% siendo muy superior al de la mezcla de los componentes iniciales de la pila que se obtuvo un índice de germinación de $48,76 \%$ el aumento del porcentaje de índice de germinación es por la conversión eficiente de la materia orgánica durante el proceso en la que se eliminan todo material fitotóxico que inhiben el crecimiento de las semillas cultivadas, el porcentaje de germinación alcanzado es un buen indicador que el grado de madurez alcanzado por el producto es el adecuado.

\section{Fósforo}

El porcentaje de fósforo expresado en forma de óxido de fosforo $\left(\mathrm{P}_{2} \mathrm{O}_{5}\right)$, en el compost fue de $0,34 \%$ el incremento de la concentración de este elemento una vez que termino el proceso es consecuencia de la perdida de materia orgánica que es mineralizada durante todo el proceso, la concentración de este elemento en la cantidad obtenida ayuda a enriquecer los suelos y mejora los cultivos mediante la absorción de este elemento en forma de fosfatos (SÁEZ. A., SÁEZ O, Andrés. Métodos para mejorar la 
calidad del compost de la fracción orgánica de los residuos sólidos. [En línea] (Tesis pregrado) (Licenciado Geólogo). Universidad Politécnica de Madrid, 2000, pág. 130).

\section{Nitrógeno}

El porcentaje de nitrógeno en el compost fue de 2,20\%, un valor superior al obtenido en varias investigaciones similares, puesto que se considera que un compost maduro presenta valores aproximados al $1 \%$ el valor alto de nitrógeno obtenido en el producto final se debe a que los materiales orgánicos iniciales que se compostaron fueron residuos ricos en nitrógeno como los residuos de poda de árboles con un porcentaje de 2,23\% y los residuos de mercado con un valor de 1,82 , puesto que materias orgánicas pobres en el contenido de nitrógeno alcanzan valores entre 0,8 y 1 $\%$ y residuos ricos en nitrógeno al madurar el compost alcanzan valores entre 2 y $3 \%$ (SÁEZ. A., SÁEZ O, Andrés. Métodos para mejorar la calidad del compost de la fracción orgánica de los residups sólidos. (Tesis) (Ldo. Geo). Universidad Politécnica de Madrid. Escuela técnica superior de ingenieros de caminos, canales y puertos, 2000, pág. 130).

\section{Potasio}

El potasio se puede obtener de diferentes fuentes de residuos orgánicos el porcentaje de potasio como Oxido de potasio $\left(\mathrm{K}_{2} \mathrm{O}\right)$ del compost fue de $1,69 \%$ este porcentaje se alcanzó puesto que los residuos orgánicos del Mercado Mayorista contenían desechos de plátano, verduras, hortalizas y restos de preparada que se comercializan en este mercado (SÁEZ. A., SÁEZ $\mathrm{O}$, Andrés. Métodos para mejorar la calidad del compost de la fracción orgánica de los residups sólidos. (Tesis) (Ldo. Geo). Universidad Politécnica de Madrid. Escuela técnica superior de ingenieros de caminos, canales y puertos, 2000, pág. 130).

\section{Olor}

El compost presentó un olor a tierra fresca de bosque, este olor característico se alcanzó debido a la ausencia de ácidos orgánicos en el producto una vez que finalizó el proceso de compostaje cuando el material orgánico inicial alcanzó su degradación biológica realizada por los organismos; un resultado semejante se obtuvo en el trabajo realizado (LÓPEZ. W, López, Wong, Wenndy, 2010, págs. 92,93)

\section{Color}

El compost presentó un color entre café oscuro a negro, como consecuencia de la transformación de la materia orgánica en sustancias 
húmicas así como también la formación de cromóforos y melanoidinas, moléculas que se conformaron una vez que el material orgánico alcanzó temperaturas termofílicas y experimento un tiempo degradativo prolongado lo cual indica la finalización del proceso, resultados que se correlacionan con los obtenidos por (IGLESIAS. E, 2014, pág. 12), en su trabajo sobre métodos y parámetros para evaluar la madurez del compost.

\section{Duración del proceso}

El tiempo necesario que debe transcurrir para obtener un producto estable depende de varios factores principalmente de la temperatura ambiente de la zona, la estación del año en la que se elabore compost, el tipo de materiales a compostar, del tamaño de partícula, el sistema utilizado entre otros (ALTAMIRANO. M \& CABRERA. C, 2006, pág. 80).

En nuestro estudio el tiempo transcurrido desde la implantación de la pila hasta la obtención del producto final fue de 224 días. El tiempo que se demoró para obtener el compost se debe en parte a la temperatura ambiente promedio del cantón Riobamba lo cual retrasa el proceso y hace que se requiera de más tiempo para la degradación completa del material compostable, la temperatura de la zona varía entre $13{ }^{\circ} \mathrm{C}$ y $15{ }^{\circ} \mathrm{C}$ según los datos meteorológicos (ESPOCH, 2015) y en parte a los residuos orgánicos vegetales de poda de árboles y palma los mismos que por contener en su estructura altas concentraciones de fibra, lignina, celulosa y hemicelulosa, compuestos que no pueden ser degradados de forma rápida sino que requieren un tiempo más prolongado para ser degradados por completo por los microorganismos aerobios (GALEA. Z, Galea, Camacho,Zesay, 2013, pág. 19).

\section{Textura}

El cambio físico notable que presentó el material una vez finalizado el proceso fue su textura la misma que era muy granular y bastante suelta, debido principalmente a la actividad microbiana que se desarrolló por un elevado contenido de materia orgánica de los materiales compostables, a los volteos del material y el control permanente del proceso. 


\section{Rendimiento}

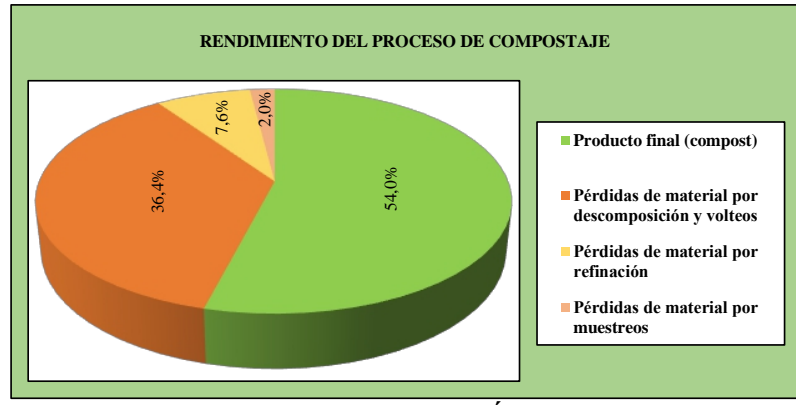

FUENTE: BRITO H. / JIMÉNEZ. S, 2015

Gráfico 6: Rendimiento del proceso

El proceso de compostaje se inició con un peso de $1000 \mathrm{~kg}$ de residuos sólidos orgánicos una vez que finalizo el proceso se obtuvo como producto (compost) $540 \mathrm{~kg}$.

Es decir, el rendimiento del proceso fue del $54,0 \%$. La pérdida de peso se dio en un $364 \mathrm{~kg}$ de pérdidas por descomposición microbiana debido de las transformaciones bioquímicas de la materia orgánica y actividades de volteo, $76 \mathrm{~kg}$ en pérdida de material por la refinación del producto para eliminar los materiales que no se degradaron totalmente durante el proceso de compostaje y que presentaron un tamaño de partícula superior a $1 \mathrm{~cm}$ y un $20 \mathrm{~kg}$ por pérdidas de material que se extrajo para realizar los análisis de los parámetros físicos y químicos utilizados para el control del proceso (TIERRA. S, 2010, pág. 19).

\section{Conclusiones y recomendaciones \\ Conclusiones}

$\checkmark \quad$ Una vez finalizado el proceso de compostaje se obtuvo $540 \mathrm{~kg}$ de compost y $460 \mathrm{~kg}$ en pérdidas de materiales.

$\checkmark \quad$ El producto obtenido mediante la técnica de biodegradación de un sistema abierto con apilamiento del material duró 224 días.

$\checkmark \quad$ El compost presentó las siguientes propiedades a través de los análisis físico-químicos, químicos y biológicos de varios parámetros entre ellos se obtuvo un 49,59 \% de materia orgánica, un $\mathrm{pH}$ de 8,85 , una conductividad eléctrica de 2,20 y un índice de germinación del 85,25\%.

\section{Recomendaciones}

$\checkmark \quad$ Para tratar los residuos orgánicos del mercado Mayorista mediante compostaje, se debe partir de materia orgánica libre de contaminantes, por lo que se recomienda una recolección selectiva en los puestos de venta y áreas de almacenamiento. 
$\checkmark \quad$ Para realizar la manipulación de forma manual de las pilas la altura de la misma no debe ser mayor a un $1,5 \mathrm{~m}$ de alto y 2,0 $\mathrm{m}$ de ancho por facilidad de manejo y para conservar la temperatura adecuada.

$\checkmark \quad$ La maquinaria que se utilice para triturar los diversos componentes orgánicos para elaborar compost debe ser la adecuada para reducir costos y tiempo operación.

$\checkmark \quad$ Los instrumentos que se utilicen para la medición de los parámetros del proceso deben ser confiables para obtener datos más reales y optimizar tiempos de medición.

$\checkmark \quad$ Para obtener información más real de la cantidad de residuos sólidos que se generan en el mercado Mayorista se recomienda clasificar y cuantificar al menos durante una semana de cada trimestre del año.

\section{Rerefences:}

1. ALTAMIRANO. M, F., \& CABRERA. C, C. (2006). ALTAMIRANO F, María ., \& CABRERA C, Carlos. Estudio comparativo para la elaboración de compost por técnica manual. (F. M. Altamirano, \& C. C. Cabrera, Edits.) Revista del Instituto de Investigaciones FIGMMG. Vol. 9, N. 17. 2006, p. 80,81 Disponible en:

http://sisbib.unmsm.edu.pe/bibvirtualdata/publicaciones/geologia/vol 9_n17/a10.pdf 2015-04-05.

2. CHÁVEZ. L. (2012). CHÁVEZ R, Luis M. Uso de desechos de camal (contenido ruminal, sangre y estiércol) en la elaboración de compost con la utilización de diferentes sustratos. (Tesis) (Ing. Agro). Ecuela Politécnica del Ejército, Departamento de Ciencias de la Vida. Sangolquí-Ecuador. 2012, pp. 12-14,15,64-68. Obtenido de http://repositorio.espe.edu.ec/bitstream/21000/7926/3/T-ESPE-IASAI-004753.pdf

3. ESPOCH. (2015). ESPOCH. Resumen climático de la Estación metereológica Espoch. Riobamba-Ecuador. 2015. Obtenido de http://www.espoch.edu.ec/index.php?action=facultades \&id=7 2015-07-15

4. FAO. (2013). FAO. Manual de compostaje del agricultor. Santiago de Chile-Chile. Obtenido de http://www.fao.org/docrep/019/i3388s/i3388s.pdf

5. GALEA. Z. (2013). GALEA C, Zesay. Ensayo de producción y caracterización de compost a partir de residuos de guacamole, poda y gallinaza. (Tesis pregrado). (Licenciada Ambiental). Universidad Pablo de Olavide, Departamento de Sistemas Físicos, Químicos y Naturales. Sevilla-España. 2013, pp. 14-25. [Consulta: 2015-05-26]. 
http://digital.csic.es/bitstream/10261/80186/1/Ensayo\%20de\%20prod ucci\%C3\%B3n.pdf.

6. GALEA. Z. (2013). GALEA C, Zesay. Ensayo de producción y caracterización de compost a partir de residuos de guacamole, poda y gallinaza. (Tesis) (Lda. Amb). Universidad Pablo de Olavide, Departamento de Sistemas Físicos, Químicos y Naturales. Sevilla-España. 2013, pp. 14, 17-20, 21, 24, 25: Disponible en: http://digital.csic.es/bitstream/10261/80186/1/Ensayo\%20de\%20prod ucci $\%$ C3\%B3n.pdf 2015-04-01.

7. GORDILLO. F, \& CHÁVEZ. E. (2010). GORDILlO, F., \& CHÁVEZ, E. Evaluación comparativa de la calidad del compost producido a partir de diferentes combinaciones de desechos agroindustriales azucareros. (Tesis) (Ing. BT). (Escuela Superior Politécnica del Litoral, Facultad de Ingenieria Mecanica y Ciencias de la Producción. 2010, Guayaquil-Ecuador, pp. 3,4.

8. IGLESIAS. E. (2014). IGLESIAS J, Emeterio. Métodos y parámetros para evaluar la madurez del compost. Istituto de Recursos Naturales y Agrobiología. Departamento de Estrés Abiótico. Salamanca-España.CSIC. 2014, pp. 9,10, 12. Obtenido de http://digital.csic.es/bitstream/10261/92881/1/3\%202\%20Unidad\%20 tematica\%204.pdf 2015-02-22

9. JARAMILLO. G, \& ZAPATA. L. (2008). JARAMILLO H, Gladis., \& ZAPATA M, Liliana M. Aprovechamiento de los residuos sólidos orgánicos en Colombia. (Tesis) (Esp. Ges. Amb). Universidad de Antioquia, Facultad de ingeniería, Departamento de Posgrados de ambiental. Antioquía-Colombia. 2008, pp. 25,27,36,37,41:

10. LÓPEZ. W. (2010). LÓPEZ W, Wenndy. Estudio del uso de residuos industriales no peligrosos a través del proceso de compostaje y su aplicación para el cultivo de maíz y frijol. (Tesis pregrado). (Maestra en Biotecnología Aplicada). Instituto Politécnico Nacional. Tlaxcala-México. 2010, pp.25-93. [Consulta: 2015-04-26].

11. LÓPEZ. W. (2010). LÓPEZ W, Wenndy. Estudio del uso de residuos industriales no peligrosos a través del proceso de compostaje y su aplicación para el cultivo de maíz y frijol. (Tesis) (Ma. BT). Instituto Politecnico Nacional. Tlaxcala-México. 2010, pp.25,26,92,93:

12. MARMOLEJO. L, F., OVIEDO. E, R., JAIMES. J, C., \& TORRES. P. (2010). MARMOLEJO, Luis F., et al. Influencia de la separación en la fuente sobre el compostaje de resuduos sólidos municipales. Agronomía Colombiana. Vol. 28, N. 2. 2010, Cali-Colombia, pp. $321-325$ Disponible en: http://www.scielo.org.co/pdf/agc/v28n2/v28n2a21 2015-04-13. 
13. OLIVEIRA DE SIQUEIRA, T. M. (2015). COMPOSTING OF MUNICIPAL SOLID WASTE IN THE STATE OF SAO PAULO (BRAZIL). Sao Paulo.

14. QUISHPE. D, \& SUQUILANDA. M. (2008). QUISHPE L, Diego., \& SUQUILANDA $V$, Manuel. Evaluación de tres mezclas de desechos biodegradables inoculados con tres dosis de microorganismos efectivos en la elaboración de compost. Cayambe-Pichincha. (Tesis) (Ing). Universidad central del Ecuador, Facultad de Ciencias Agricolas. Cayambe-Ecuador, 2008, pp. 3-10.

15. SANABRIA. M. (2013). SANABRIA C, María F. Proceso de validación en manejo y uso de residuos orgánicos. Bogotá-Colombia. 2013, pp. 10,11,23,27.

16. TIERRA. S. (2010). TIERRA T, Susana del P. Evaluación nutrimental de compost proveniente de cuatro combinaciones de desechos orgánicos frente a la aplicación de eco-abonaza en el cultivo de lechuga (lactuca sativa). (Tesis pregrado) (Ingeniero Agrónomo). Escuela Superior Politécnica de Chimborazo, Facultad de Recursos Naturales, Escuela de ingeniería Agronómica, Riobamba-Ecuador. 2010, pp. 16-21. 